\title{
Valeur nutritive des ensilages combinés de fientes de volailles et de grignons d'olives. I. Influence de la durée d'accumulation des fientes et des proportions respectives de fientes et de grignons sur la composition chimique et les caractéristiques fermentaires des ensilages
}

\author{
A Nefzaoui \\ INRA de Tunisie, laboratoire de nutrition animale, Ariana 2080, Tunisie
}

(Reçu le 19 décembre 1990; accepté le 25 mars 1991)

\begin{abstract}
Résumé - Les élevages avicoles d'une part et les industries oléicoles d'autre part, produisent de grandes quantités de fientes de volailles et de grignons d'olive en Tunisie. Les fientes sont très riches en MAT (26-32\% de la MS) dont une grande partie sous forme soluble (59-70\% de l'azote total) et ammoniacale $(36,4-44,4 \%$ de l'azote total). Les grignons ne contiennent pratiquement pas d'azote sous forme soluble ou ammoniacale, mais sont très riches en constituants pariétaux $(55,7 \%$ NDF, $45 \%$ ADF et $32 \% A D L$ ). Le mélange de ces 2 résidus peut constituer une source d'aliment pour le bétail dans certains pays méditerranéens. La dégradation rapide des fientes nous a conduit à envisager la possibilité d'ensiler ces 2 résidus. Dans un premier temps, nous avons étudié l'effet de la durée d'accumulation des fientes $(7,14,21,28,35$ et 43 j) sur leur composition chimique. Puis, nous avons cherché, en combinant des grignons avec des fientes ayant différentes durées d'accumulation et dans des proportions différentes $(33,44,57,75$ et $100 \%$ de fientes), à déterminer les limites maximales permettant d'avoir des ensilages de qualité acceptable. II apparaît que la durée d'accumulation des fientes ne doit pas dépasser 3 semaines, si on veut éviter la dégradation excessive de la MO. La part des fientes, sur la base de la MS, ne doit pas non plus dépasser $40-45 \%$ dans le mélange, seuil au-dessus duquel la qualité de conservation est compromise ( $\mathrm{pH}$ élevé, forte teneur en acide butyrique).
\end{abstract}

ensilage / fiente de volaille / grignon d'olive

Summary - Nutritive value of combined laying hen excreta and olive cake sllages. I. Effect of storage time and laying hen excreta levels on chemical composition and fermentation characteristics of silages. Large amounts of laying hen excreta and olive cakes are available in Tunisia. The former has a high crude protein content (26-32\% DM). This nitrogen is mainly soluble (59-70\% of total nitrogen) or ammoniacal (36.4-44.4\% total nitrogen). The olive cake does not contain either soluble or ammonia nitrogen, but high levels of NDF (55.4\%), ADF (45\%) and ADL (32\%). Mixing these 2 by-products may constitute an animal feed resource for many mediterranean countries. Ensiling these by-products may be a solution for limiting the rapid degradation of laying hen organic excretory matter. The effect of storage time $(7,14,21,35$ and 43 d) on chemical composition of laying hen excreta was investigated (table l). The effects of storage time and laying hen excreta levels (33, $44,57,75$ and $100 \%$ on a dry matter basis) were examined in order to determine the optimal limits 
for obtaining good quality silages (table II). Storage time $>3$ wk had a negative effect on silage quality (table III). Moreover, 55\% of laying hen excreta in the mixture seemed to be the upper limit for good quality silages.

\section{silages / laying hen excreta / olive cake}

\section{INTRODUCTION}

Les déchets animaux peuvent représenter un vaste réservoir d'éléments nutritifs, surtout pour les ruminants. Dans la plupart des pays, les excréments et particulièrement ceux de la volaille, sont facilement "collectables".

L'idée de recycler les fientes de volailles dans l'alimentation des animaux est relativement récente. Les fientes peuvent être utilisées comme source de protéines et de minéraux (El-Sabban et al, 1970; Fontenot et Webb, 1974; Cullison et al, 1976; Muller, 1980).

Les fientes sont composées de résidus indigestibles de la ration, de composés métaboliques d'origine endogène et de cellules et microorganismes du tractus digestif de la volaille. Leur composition chimique varie alors selon la composition de la ration, le gaspillage de l'aliment et la quantité de plumes qu'elles contiennent, ainsi que de l'environnement (Evans et al, 1978; Muller, 1980).

Les fientes "fraîches" ont une teneur en matière sèche (MS) de 13 à $26 \%$ et en matières azotées totales (MAT) de 20 à $45 \%$ dont 24 à $41 \%$ sont sous la forme d'acides aminés (Bhattacharya et Taylor, 1975).

Les fientes sont très riches en minéraux, et en particulier en calcium et en phosphore, respectivement 1,9 et $1,7 \%$ de la MS des fientes de poulets et 7,5 et $2,6 \%$ de la MS des fientes de pondeuses (Muller, 1980).
La décomposition rapide de la matière organique (MO) des fientes est sous l'influence directe de l'environnement, surtout l'humidité et les températures élevées. Ces dernières activent la vitesse de dégradation enzymatique de l'acide urique et de l'urée en ammoniac. C'est ainsi que la teneur en MAT passe de 30 à $25 \%$ et à $18,3 \%$ de la MS après respectivement 7 , 49 et $98 j$ d'accumulation sous les batteries (Flegal et al, 1972). Chang et al (1975) ont montré que la teneur des fientes en ammoniac était multipliée par 6 , seulement $3 \mathrm{~h}$ après la collecte.

Les fientes devront donc être traitées rapidement pour éviter leur décomposition rapide. Le séchage, technique largement utilisée, est coûteux et entraîne des pertes d'azote (Muller, 1980). Une alternative consiste en leur ensilage avec des résidus de récolte. Cette technique de conservation, sans doute moins coûteuse, limite les pertes d'azote si les processus fermentaires se déroulent convenablement.

La Tunisie dispose de grandes quantités de fientes qui posent de sérieux problèmes d'environnement. De plus, l'industrie oléicole laisse un résidu abondant, les grignons d'olive, de nature lignocellulosique et de faible valeur nutritive.

Nous nous proposons d'étudier la possibilité d'ensiler ensemble ces 2 résidus, dans l'objectif de leur utilisation ultérieure dans l'alimentation des ruminants. Dans cette contribution, nous traiterons :

- de l'effet de la durée d'accumulation des fientes sous les batteries sur leur composition chimique, 
- de l'influence de la proportion et de la durée d'accumulation des fientes sur la composition chimique et les caractéristiques fermentaires des ensilages de grignons d'olive et de fientes de pondeuses.

\section{MATÉRIEL ET MÉTHODES}

\section{Les sous-produits}

Les fientes provenaient d'un élevage de pondeuses de la souche Sexa-Line, dont la consommation journalière était de $110 \mathrm{~g}$ de farine commerciale et $200 \mathrm{~g}$ d'eau par jour. La température du poulailler était de 18 à $20^{\circ} \mathrm{C}$.

Le grignon d'olive a été prélevé dans des huileries fonctionnant à pression. Après son épuisement à l'hexane, il a été tamisé pour soustraire les débris d'endocarpes. II s'agit donc de grignon épuisé tamisé (GET). II contenait en moyenne $90,6 \%$ de matière sèche (MS) et en $\%$ de la MS, $10,75 \%$ de matières azotées totales (MAT), 3,5\% de matières grasses (MG), $13,32 \%$ de cellulose brute (CB), $55,70 \%$ de constituants pariétaux (NDF), $45 \%$ de lignocellulose (ADF) et $32 \%$ de lignine ( $A D L$ ). L'azote soluble et ammoniacal étaient à l'état de traces.

\section{Prélèvements des fientes et ensilages}

Les prélèvements de fientes aux fins d'analyses et d'ensilage ont été effectués après des durées d'accumulation sous les batteries (ou âge des fientes) de $7,14,21,28,35$ et $43 \mathrm{j}$.

Les ensilages ont été réalisés en triple dans des microsilos en verre de 1,5 I de capacité. Les fientes de différents âges ont été mélangées intimement avec du grignon (GET) mélassé à $20 \%$. L'addition de la mélasse procurait les sucres nécessaires à la fermentation lactique. Differentes proportions de fientes et de grignon ont été testées. Sur la base de la MS, la part des fientes a été de $33,44,57,75$ et $100 \%$, soit $60,70,80$, 90 et 100 en $\%$ des produits bruts. La durée de conservation a été de 3 mois.

\section{Mesures et méthodes analytiques}

La composition chimique des fientes a été effectuée, juste après les prélèvements sur les produits frais (MS, MAT, azote soluble, azote ammoniacal, $\mathrm{pH}$ ) ou lyophilisés (matières minérales, NDF, ADF, ADL, CB).

A l'ouverture des silos, les caractéristiques fermentaires des ensilages $(\mathrm{pH}$, acides organiques, ammoniac, azote soluble) ont été mesurées sur l'extrait à l'eau distillée. Les autres caractéristiques chimiques des ensilages ont été effectuées sur des échantillons lyophilisés.

La MS a été obtenue après sèchage à $105^{\circ} \mathrm{C}$ dans une étuve ventilée et corrigée pour les produits volatils selon Schoch (1949). Les acides organiques ont été déterminés par distillation suivant la méthode de Flieg décrit par Vervack (1983) et l'azote total $\left(N_{t}\right)$ et soluble $\left(\mathrm{N}_{\mathrm{s}}\right)$ par la méthode de Kjeldahl. L'azote ammoniacal $\left(\mathrm{N}-\mathrm{NH}_{3}\right)$ est obtenu par distillation après neutralisation avec du $\mathrm{MgO}$. Les matières protéiques (MP) ont été dosées par la méthode Kjeldahl après leur précipitation au $\mathrm{CuSO}_{4}$. La cellulose brute (cellulose Weende) a été déterminée après une hydrolyse acide suivie d'une hydrolyse basique. Les constituants pariétaux (NDF, $A D F, A D L)$ ont été déterminés sur des échantillons lyophilisés et moulus $(1 \mathrm{~mm})$ selon la méthode de Goering et Van Soest (1970).

\section{RÉSULTATS}

\section{Influence de la durée d'accumulation sur la composition chimique des fientes}

La teneur en matière sèche (MS) augmente légèrement jusqu'à la $3^{\theta}$ semaine et diminue ensuite progressivement pour atteindre la valeur de $22,7 \%$ à 43 j (tableau I).

La teneur en matière organique $(\mathrm{MO}) \mathrm{dj-}$ minue progressivement de $71 \%$ à $7 \mathrm{j}$, à $63 \%$ à $43 \mathrm{j}$. Les matières protéiques totales demeurent stables durant les 3 pre- 
Tableau I. Évolution de la composition chimique des fientes de poules pondeuses en fonction de la durée d'accumulation, \% MS.

\begin{tabular}{|c|c|c|c|c|c|c|}
\hline Durées d'accumulations ( $(j)$ & 7 & 14 & 21 & 28 & 32 & 43 \\
\hline $\begin{array}{l}\text { MS, \% } \\
\mathrm{pH}\end{array}$ & $\begin{array}{r}29,8 \\
7,0\end{array}$ & $\begin{array}{r}30,3 \\
7,2\end{array}$ & $\begin{array}{r}30,3 \\
7,3\end{array}$ & $\begin{array}{r}28,9 \\
7,7\end{array}$ & $\begin{array}{r}28,6 \\
7,9\end{array}$ & $\begin{array}{r}27,7 \\
8,0\end{array}$ \\
\hline $\begin{array}{l}\text { MAT }(\%), M S \\
N-N H_{3}(\%), N \text { total } \\
\mathrm{N} \text { soluble } \% \mathrm{~N} \text { total } \\
\text { MP, \% MS }\end{array}$ & $\begin{array}{l}26,2 \\
42,2 \\
64,5 \\
17,8\end{array}$ & $\begin{array}{l}32,1 \\
38,6 \\
60,3 \\
17,9\end{array}$ & $\begin{array}{l}29,58 \\
42,1 \\
62,9 \\
17,5\end{array}$ & $\begin{array}{l}27,7 \\
44,4 \\
69,9 \\
14,5\end{array}$ & $\begin{array}{l}31,7 \\
36,4 \\
58,8 \\
13,8\end{array}$ & $\begin{array}{l}29,4 \\
43,1 \\
64,4 \\
13,6\end{array}$ \\
\hline $\begin{array}{l}\text { NDF, \% MS } \\
\text { ADF, \% MS } \\
\text { ADL, \% MS }\end{array}$ & $\begin{array}{r}37,9 \\
20,0 \\
3,4\end{array}$ & $\begin{array}{r}38,1 \\
19,2 \\
3,1\end{array}$ & $\begin{array}{r}39,8 \\
19,2 \\
3,4\end{array}$ & $\begin{array}{r}39,3 \\
20,6 \\
3,8\end{array}$ & $\begin{array}{r}37,0 \\
20,9 \\
4,4\end{array}$ & $\begin{array}{r}40,6 \\
21,5 \\
3,9\end{array}$ \\
\hline $\begin{array}{l}\text { Cendres totales, } \% \text { MS } \\
\text { Cendres insolubles, \% MS }\end{array}$ & $\begin{array}{r}29,0 \\
5,7\end{array}$ & $\begin{array}{r}30,2 \\
5,9\end{array}$ & $\begin{array}{r}31,7 \\
8,9\end{array}$ & $\begin{array}{r}33,7 \\
7,4\end{array}$ & $\begin{array}{r}34,7 \\
9,2\end{array}$ & $\begin{array}{r}36,7 \\
9,0\end{array}$ \\
\hline $\begin{array}{l}\mathrm{P}, \% \mathrm{MS} \\
\mathrm{Ca}, \% \mathrm{MS} \\
\mathrm{Na}, \% \mathrm{MS} \\
\mathrm{K}, \% \mathrm{MS} \\
\mathrm{Mg}, \% \mathrm{MS} \\
\mathrm{Fe}, \% \mathrm{MS} \\
\mathrm{Cu}, \mathrm{ppm} \\
\mathrm{Zn}, \mathrm{ppm}\end{array}$ & $\begin{array}{r}1,47 \\
6,77 \\
0,35 \\
2,29 \\
1,39 \\
0,12 \\
39 \\
290\end{array}$ & $\begin{array}{c}1,47 \\
6,81 \\
0,23 \\
2,48 \\
1,51 \\
0,11 \\
47 \\
396\end{array}$ & $\begin{array}{c}1,48 \\
6,47 \\
0,26 \\
2,59 \\
1,56 \\
0,04 \\
24 \\
312\end{array}$ & $\begin{array}{c}1,52 \\
7,42 \\
0,27 \\
2,77 \\
2,77 \\
0,04 \\
25 \\
335\end{array}$ & $\begin{array}{c}1,36 \\
6,69 \\
0,22 \\
2,51 \\
2,51 \\
0,04 \\
36 \\
340\end{array}$ & $\begin{array}{c}1,58 \\
7,06 \\
0,23 \\
3,00 \\
3,00 \\
0,04 \\
34 \\
325\end{array}$ \\
\hline
\end{tabular}

mières semaines et diminuent par la suite, pour avoir une valeur de $13,6 \%$ à $43 \mathrm{j}$. Le $\mathrm{pH}$, par contre, augmente progressivement de 7,03 à $7 \mathrm{j}$, à 7,99 à $43 \mathrm{j}$.

\section{Influence de la durée d'accumulation des fientes sur la composition chimique et les caractéristiques fermentaires des ensilages}

La durêe d'accumulation des fientes agit significativement sur la teneur en MS et sur le $\mathrm{pH}$ des ensilages (tableau II). Elle diminue significativement les teneurs en matières azotées totales (MAT) et en acide acétique. Elle n'a pas d'influence significative sur les teneurs en acides butyrique et lactique et sur les constituants pariétaux.

Influence de la proportion de fientes sur la composition chimique et les caractéristiques fermentaires des ensilages

Les proportions croissantes de fientes agissent significativement sur tous les paramètres de composition chimique à l'exception de la cellulose et de l'hémicellulose (tableau II!). 
Tableau II. Influence de la durée d'accumulation des fientes sur la composition chimique et les caractéristiques fermentaires des ensilages (en \% de la matière sèche).

\begin{tabular}{lcccccccc}
\hline Durées d'accumulations (j) & 7 & 14 & 21 & 28 & 35 & 43 & ESM & \\
\hline MS, \% & 39,7 & 43,1 & 41,7 & 41,6 & 40,9 & 43,1 & 0,60 & $*$ \\
pH & 5,85 & 6,08 & 6,42 & 6,47 & 6,28 & 6,54 & 0,15 & $*$ \\
MAT, \% MS & 22,5 & 22,2 & 22,1 & 21,8 & 20,2 & 21,3 & 0,38 & $*$ \\
N-NH ${ }_{3} \%$ N total & 30,9 & 43,3 & 35,3 & 32,9 & 34,4 & 31,2 & 2,38 & $*$ \\
N soluble \% N total & 55,8 & 62,1 & 56,6 & 53,2 & 59,4 & 53,5 & 3,63 & NS \\
NDF, \% MS & 38,1 & 36,6 & 37,6 & 38,9 & 38,8 & 37,7 & 0,78 & NS \\
ADF, \% MS & 30,0 & 27,7 & 29,0 & 29,2 & 30,6 & 29,3 & 0,55 & NS \\
ADL, \% MS & 16,1 & 15,8 & 15,8 & 16,4 & 17,4 & 16,6 & 0,42 & NS \\
Acide acétique, \% MS & 5,6 & 4,2 & 4,3 & 3,5 & 4,4 & 3,4 & 0,38 & $*$ \\
Acide butyrique, \% MS & 2,1 & 3,3 & 3,0 & 2,7 & 3,2 & 2,5 & 0,39 & NS \\
Acide lactique, \% MS & 5,6 & 6,1 & 5,3 & 4,6 & 4,2 & 5,4 & 0,47 & NS \\
& & & & & & & & \\
\hline
\end{tabular}

ESM : erreur standard de la moyenne; NS : effet non significatif; * : effet significatif $(P<0,05) ;{ }^{* *}$ : effet très significatif $(P<0,01)$.

Tableau III. Influence de la proportion de fientes sur la composition chimique et les caractéristiques fermentaires des ensilages, \% MS.

\begin{tabular}{|c|c|c|c|c|c|c|c|}
\hline Proportions de fientes & 33 & 44 & 57 & 75 & 100 & $E S M$ & \\
\hline $\begin{array}{l}\mathrm{MS}, \% \\
\mathrm{pH} \\
\mathrm{MAT}, \% \mathrm{MS} \\
\mathrm{N}-\mathrm{NH}_{3}, \% \mathrm{~N} \text { total } \\
\mathrm{N} \text { soluble \% } \mathrm{N} \text { total } \\
\text { Acide acétique, \% MS } \\
\text { Acide butyrique, \% MS } \\
\text { Acide lactique, \% MS }\end{array}$ & $\begin{array}{c}54,8 \\
5,54 \\
15,9 \\
18,3 \\
45,4 \\
2,1 \\
0,1 \\
7,9\end{array}$ & $\begin{array}{c}49,3 \\
5,60 \\
18,0 \\
24,4 \\
49,5 \\
2,5 \\
0,3 \\
8,4\end{array}$ & $\begin{array}{c}41,9 \\
6,03 \\
20,8 \\
33,3 \\
58,3 \\
4,1 \\
0,7 \\
7,9\end{array}$ & $\begin{array}{c}33,7 \\
6,93 \\
25,9 \\
46,3 \\
65,5 \\
5,6 \\
6,5 \\
1,3\end{array}$ & $\begin{array}{c}28,8 \\
7,29 \\
28,3 \\
51,1 \\
65,0 \\
6,8 \\
6,5 \\
0,5\end{array}$ & $\begin{array}{l}0,55 \\
0,14 \\
0,35 \\
2,17 \\
3,31 \\
0,34 \\
0,35 \\
0,43\end{array}$ & $\begin{array}{l}* * * \\
* * * \\
* * * \\
* * * \\
* * * \\
* * * \\
* * * \\
* * *\end{array}$ \\
\hline
\end{tabular}

ESM : erreur standard de la moyenne; $\left(^{* * *}\right)$ effet très hautement significatif $(P<0,001)$.

\section{DISCUSSIONS}

Les compositions chimiques des fientes étudiées sont comparables à celles rapportées dans la littérature (Flegal et al, 1972; Muller, 1980). Les fientes sont caractérisées par une teneur élevée en minéraux
(30\% de la MS) et en MAT $(26-30 \%$ de la MS). Leur composition est fortement influencée par la durée d'accumulation sous les batteries. Quand cette dernière augmente, les teneurs en MO et en matières protéiques diminuent et le $\mathrm{pH}$ augmente. Cette évolution témoigne de la dégradation 
rapide de certains constituants organiques des fientes. Ces constatations sont en accord avec celles de Flegal et al (1972) et Chang et al (1975). Ces derniers observent une perte en MAT sous forme d'ammoniac. Ils constatent une diminution des MAT de 30 et $25 \%$ de la MS, respectivement après 7 et 41 j d'accumulation.

Dans les conditions de nos essais, nous pouvons considérer qu'une durée d'accumulation de 3 semaines serait une limite à ne pas dépasser, si nous souhaitons garder le maximum d'éléments nutritifs dans les fientes.

Dans cet essai, les températures n'ont guère dépassé les $20^{\circ} \mathrm{C}$ et il est certain que la dégradation des fientes serait encore plus intense durant la saison estivale en Tunisie.

La teneur en cendres totales a varié de 29 à $37 \%$ de la MS, respectivement après 7 et 43 j d'accumulation. Des valeurs allant de 8 à $34 \%$ ont été cités par plusieurs auteurs (ie Bhattacharya et Fontenot, 1965; El-Sabban et al, 1970). Ces auteurs assurent que les fientes constituent une bonne source de $\mathrm{Ca}$ et de $\mathrm{P}$ pour les animaux. Nos données indiquent que cette source nécessite une rectification avant son utilisation pour les ruminants, car le rapport $\mathrm{Ca} / \mathrm{P}$ est de 4,5 en moyenne. De plus, les teneurs relativement élevées en cuivre doivent être prises en considération, particulièrement pour les ovins.

L'ensilage des fientes de volailles de différentes durées d'accumulation, avec des proportions décroissantes de grignon épuisé tamisé, nous permet de constater une baisse de la qualité des ensilages si la durée d'accumulation dépasse 21 j $(\mathrm{pH}$ plus élevé, teneur plus faible en acide lactique). De plus, l'ensilage avec une proportion de fientes supérieure à $55 \%$ dans le mélange serait à déconseiller, au vu de l'augmentation spectaculaire de la teneur en acide butyrique, du $\mathrm{pH}$ et de l'ammo- niac et de la diminution de la teneur en acide lactique. Le $\mathrm{pH}$ des ensilages passe de 5,54 à 7,29, quand la teneur en MS des ensilages passe de 55 à $29 \%$. Caswell et al (1978) ont évalué l'effet de la MS sur la valeur nutritive et bactériologique des ensilages de fientes. Leurs résultats montrent que $60 \%$ de MS assurent une bonne conservation, en diminuant le $\mathrm{pH}$ de 7,61 à 5,72 et en produisant suffisamment d'acide lactique $(2,83 \%)$. Fontenot et Webb (1975) trouvent que la fermentation lactique des fientes serait optimaie à $60 \%$ de MS.

Enfin, l'évaluation de la réussite de ce genre d'ensilage ne peut pas être abordée tout à fait selon les normes classiquement établies pour les fourrages, car l'apport initial des fientes en matières azotées solubles et ammoniacales est très élevé.

\section{CONCLUSIONS}

La durée d'accumulation agit négativement sur la composition chimique des fientes et se traduit par une dégradation de la MO et des matières azotées en particulier. Dans les conditions de nos essais, une durée d'accumulation supérieure à 3 semaines serait préjudiciable à la valeur nutritive de ce résidu. Pour les conditions tunisiennes et surtout durant les chaleurs estivales, cette durée doit être encore plus réduite, à cause des températures élevées qui règnent dans les bâtiments d'élevage.

La technique d'ensilage se prête bien à la préservation de la qualité nutritionnelle des fientes et des grignons, à condition d'avoir assez de sucres fermentescibles et une teneur en MS adéquate dans les silos (environ 55\%). Cette teneur en MS est déterminée indirectement par le rapport fiente/grignon. Sur la base de la MS, la part des fientes ne doit pas dépasser $55 \%$, seuil au-dessus duquel la qualité de conservation du mélange est compromise $(\mathrm{pH}$ élevé, teneur élevée en acide butyrique). 
Cependant, des essais de palatabilité, de digestibilité et surtout de l'efficacité digestive et métabolique de cette source azotée doivent être effectués pour préciser les proportions optima de fientes et de grignon et évaluer la valeur nutritive de ces mélanges.

\section{RÉFÉRENCES}

Bhattacharya AN, Fontenot JP (1965) Utilization of different levels of poultry litter nitrogen by sheep. J Anim Sci 24, 1174-1178

Bhattacharya AN, Taylor JC (1975) Recycling animal vaste as a feedstuff. A review. J Anim Sci 41, 1438-1457

Caswell LF, Fontenot JP, Webb KE (1978) Fermentation and utilization of broiler litter ensiled at different moisture levels. J Anim Sci 46, 547-561

Chang TS, Currigan DJ, Linden E (1975) Ammonia content of caged layer excreta. Res Rep 269, Michigan State Univ Agric Exp Stn East Lansing,

Cullison $A E$, McCampbell $H C$, Cunnigham $A C$, Lowrey RC, Warren EP, McLendon BD, Sherwood DH (1976) Use of poultry manures in steers finishing rations. J Anim Sci 42, 219-228

El Sabban FF, Bratzier JW, Long TA, Frear DEH, Gentry RF (1970) Value of processed poultry waste as a feed for ruminants. $J$ Anim Sci 31, 107-111

Evans E, Moran Jr ET, McLeod GK, Turner EM (1978) Laying hen excreta as a ruminant feedstuffs. II. Preservation and acceptability of wet excreta by sheep. J Anim Sci 46, 527 534

Flegal CJ, Sheppard CC, Dorin DA (1972) The effect of continuous recycling and storage on nutriment quality of dehydrated poultry waste. In: Proc Cornell Agric Waste Manage Conf, Syracuse, NY, 295-300

Fontenot JP, Webb Jr KE (1974) The value of animal waste by feeding for ruminants. Feedstuffs 46, 30-31

Fontenot JP, Webb Jr KE (1975) Ensiled broiler litter with different moisture levels. J Anim Sci 40, 200 (abstract)

Goering HK, Van Soest PJ (1970) Forage fiber analyses. Agriculture Handbook $n^{\circ} 379$. Agriculture Research Service. United States Department of Agriculture

Muller ZO (1980) Feed from animal wastes: state of knowledge. FAO - Animal Prod Health Paper 18, 190

Schoch W (1949) Die bei der Trocknung von Silage proben in trockenschrank auftretende verluste an flüchigen saüren und basen. Mitteilungen aus dem Gebiet des Lebensmitteluntersuchung und Hygien 4, 170

Vervack W (1983) Analyse des aliments. Méthodes courantes d'analyse. Laboratoire de Biochimie de la Nutrition. Université Catholique de Louvain 